Revue d'histoire de l'Amérique française

7S REVUE D.HISTOIRE DE L'AMÉRIQUE FRANÇAISE

\title{
L'histoire sociale au Québec. Réflexion sur quelques paradoxes
}

\section{Gérard Bouchard}

Volume 51, numéro 2, automne 1997

Les pratiques de l'histoire de l'Amérique française depuis 50 ans

URI : https://id.erudit.org/iderudit/305648ar

DOI : https://doi.org/10.7202/305648ar

Aller au sommaire du numéro

\section{Éditeur(s)}

Institut d'histoire de l'Amérique française

\section{ISSN}

0035-2357 (imprimé)

1492-1383 (numérique)

Découvrir la revue

\section{Citer cet article}

Bouchard, G. (1997). L'histoire sociale au Québec. Réflexion sur quelques paradoxes. Revue d'histoire de l'Amérique française, 51(2), 243-269. https://doi.org/10.7202/305648ar

\section{Résumé de l'article}

L'auteur propose une réflexion autour de certains paradoxes auxquels a donné lieu l'évolution de l'histoire sociale au Québec depuis quelques décennies. Ces paradoxes ont trait aux éléments de continuité et de discontinuité par rapport à ce que l'histoire sociale voulait être à l'origine, à son caractère très spécialisé, à la grande fragmentation des thèmes et au déclin de la synthèse, au problème de raccordement entre micro et macrohistoire, au statut marginal de la réflexion critique et épistémologique, etc. Une grande partie de l'argumentation s'appuie sur une statistique des articles et notes de recherches publiés dans la RHAF entre 1962 et 1996.

L'auteur évoque les importants progrès réalisés au cours des dernières décennies. Il signale aussi des carences, des difficultés et des défis auxquels l'histoire sociale québécoise est présentement confrontée et il suggère quelques voies de réflexion pouvant conduire à une nouvelle prise de conscience et, peut-être, à une réorientation de certains aspects de la pratique historienne.
Tous droits réservés @ Institut d'histoire de l'Amérique française, 1997
Ce document est protégé par la loi sur le droit d'auteur. L'utilisation des services d'Érudit (y compris la reproduction) est assujettie à sa politique d'utilisation que vous pouvez consulter en ligne.

https://apropos.erudit.org/fr/usagers/politique-dutilisation/ 


\title{
L'HISTOIRE SOCIALE AU QUÉBEC RÉFLEXION SUR QUELQUES PARADOXES
}

\author{
GÉRARD BOUCHARD ${ }^{1}$ \\ Institut interuniversitaire de recherches sur les populations \\ Université du Québec à Chicoutimi
}

\section{RÉSUMÉ}

L'auteur propose une réflexion autour de certains paradoxes auxquels a donné lieu l'évolution de l'histoire sociale au Québec depuis quelques décennies. Ces paradoxes ont trait aux éléments de continuité et de discontinuité par rapport à ce que l'histoire sociale voulait être à l'origine, à son caractère très spécialisé, à la grande fragmentation des thèmes et au déclin de la synthèse, au problème de raccordement entre micro et macrohistoire, au statut marginal de la réflexion critique et épistémologique, etc. Une grande partie de l'argumentation s'appuie sur une statistique des articles et notes de recherches publiés dans la RHAF entre 1962 et 1996.

L'auteur évoque les importants progrès réalisés au cours des dernières décennies. Il signale aussi des carences, des difficultés et des défis auxquels l'histoire sociale québécoise est présentement confrontée et il suggère quelques voies de réflexion pouvant conduire à une nouvelle prise de conscience et, peut-être, à une réorientation de certains aspects de la pratique historienne.

\section{ABSTRACT}

The author offers a reflection about some paradoxes to which the evolution of social history in Québec has given rise. These paradoxes pertain to the elements of continuity and discontinuity in regard to what social history intended to be at the outset, to its high level of specialization, to the fragmentation of the field as well as the waning of synthesis, the disconnexion between micro- and macro-history, the rather marginal status of epistemology, and so on. A substantial part of the argumentation draws on a quantitative survey of the articles and research notes published in the RHAF between 1962 and 1996.

The paper underlines major advances made in the last decades. It also points to some weaknesses, problems and challenges that social history in Québec is faced with. Lines of thoughts likely to spark a new consciousness, if not a reorientation of part of the field, are set forth.

1. Nous remercions nos collègues José Igartua, Yvan Lamonde et Ronald Rudin qui ont aimablement relu une version antérieure de ce texte et formulé de très utiles commentaires. Nous avons aussi tiré grand profit d'une conversation avec Fernande Roy. 
Trois voies s'offraient à cet essai sur l'évolution et l'état présent de l'histoire sociale au Québec. La première invitait à un survol de type «inventaire», faisant état des principales productions. La deuxième consistait en une revue théorique et méthodologique de l'ensemble des thèmes, des finalités, des démarches, des directions de recherche ${ }^{2}$. La troisième voie était celle de l'essai, c'est-à-dire une réflexion plus personnelle sur quelques aspects de l'histoire sociale, telle qu'elle s'est déployée au Québec au cours des dernières décennies. C'est cette dernière voie que nous avons choisie, mais sans négliger tout à fait les deux autres, comme on pourra le voir ${ }^{3}$. Concrètement, notre essai prendra la forme d'un commentaire autour de sept paradoxes.

I - Chacun aujourd'hui, au Québec, a le sentiment que l'histoire sociale s'est accomplie, qu'elle a réformé en profondeur l'ancienne pratique historienne suivant le modèle proposé par les pionniers (français surtout) des années 1940 et 1950. D'une façon ou d'une autre, la grande majorité des historiens d'ici s'en réclament au moins implicitement, elle a pénétré l'enseignement, les revues, les colloques; elle s'est institutionnalisée. Néanmoins, lorsqu'on retourne aux textes fondateurs, on note qu'au fil des ans, ce qu'on nous permettra d'appeler le programme originel de l'histoire sociale (infra) a subi plusieurs transformations. L'histoire sociale québécoise marque donc une discontinuité, une différenciation qui doit retenir l'attention, non pas parce qu'elle fait problème en ellemême, mais parce que l'évolution et les réaménagements qu'elle atteste ne semblent avoir suscité ni prise de conscience ni discussion, comme s'ils étaient simplement passés inaperçus.

II - Le vocable social accolé à la science historique voulait affirmer une réorientation générale qui plaçait au centre de l'analyse les acteurs collectifs (notamment les classes), les rapports sociaux

2. Pour une revue historiographique de ce genre, à l'échelle du Canada, voir JeanClaude Robert, «La recherche en histoire du Canada», International Journal of Canadian Studies/Revue internationale d'études canadiennes, 1-2 (printemps/automne 1990): 11-33. À propos du Québec, on consultera le bilan déjà proposé par Fernand Ouellet, «La modernisation de l'historiographie et l'émergence de l'histoire sociale», Recherches sociographiques, 26,1-2 (1985): 11-83.

3. Notre réflexion porte sur l'histoire sociale au Québec mais, en divers points, nous serons amené à déborder ce cadre pour embrasser l'ensemble du champ de la science historique. Ce débordement s'est révélé inévitable, étant donné le flou qui entoure présentement la définition même de l'histoire sociale. Par ailleurs, afin d'alléger le texte, les références bibliographiques ont été réduites au minimum. Comme il arrive toujours dans un essai de ce genre, nous avons sans doute commis des oublis; les auteurs concernés voudront bien nous le pardonner. On notera qu'au cours du texte, et pour fins de commodité seulement, la forme masculine est souvent employée en un sens générique. 
(conflictuels et autres), l'évolution des clivages et des inégalités. Lorsqu'on fait le compte au Québec, on s'aperçoit que ces thèmes ne sont pas vraiment prédominants (ils sont plutôt en déclin) et ce, au moment même où l'histoire sociale a achevé d'étendre partout sa référence.

III - L'un des premiers objectifs de cette histoire sociale était de recentrer l'analyse pour y intégrer tous les acteurs (la masse, les anonymes) et se mettre ainsi en position de parler au nom du plus grand nombre. Cet objectif très novateur, très riche, a été pleinement atteint. Mais pour y arriver, la plus grande partie de la science historique a dû se spécialiser, modifier ses procédés, se faire plus technique, transformer son écriture. Résultat: cette histoire qui se voulait plus universelle (ou plus démocratique?) dans ses conceptions n'est plus guère lue et commentée que par les gens du métier, alors que les anciennes biographies des représentants des élites faisaient les délices du public cultivé, sinon du grand public.

IV - Les pionniers de l'histoire sociale combattaient l'ancienne méthode historique qui émiettait le collectif et ses évolutions complexes dans une myriade de faits et de dates. Avec la nouvelle histoire, l'heure était désormais à la recherche des articulations, des interactions, de la synthèse: histoire sociale, histoire totale, disaiton. Or, quelques décennies plus tard, à l'étape de la maturité, la pratique historique n'a jamais été aussi fragmentée. Hormis quelques initiatives (par ailleurs très heureuses), la synthèse est devenue un genre pratiquement délaissé.

V - L'histoire sociale — qui ne l'a pas lu ou entendu cent fois? - part de l'actuel. Elle y puise les préoccupations, les questions qui initient sa démarche et guident son itinéraire dans le passé. Le praticien de l'histoire sociale, assure-t-on, est à sa manière un spécialiste du présent: il professe (avec raison) que, pour mieux le comprendre, ce dernier doit être inséré dans les tendances, dans les trames de la longue ou moyenne durée. Mais c'est précisément depuis que l'histoire sociale québécoise s'est ainsi convertie à l'actuel qu'elle semble s'être détournée des grandes questions qui agitent notre temps. Cette remarque vaut aussi pour la science historique dans son ensemble.

VI - Consciente des limites de ses procédés, l'histoire sociale a renoncé aux canons traditionnels de l'objectivité et à l'ambition de se constituer en science exacte, sur le modèle du scientisme du siècle dernier. Elle sait que son savoir est un construit sans cesse à refaire, un produit d'hypothèses, de points de vue, de paris sur 
le «réel», de paradigmes; elle est devenue relativiste. Et pourtant, au Québec, elle semble peu préoccupée de discuter et de remettre en cause ses productions, ses procédés, ses prémisses.

VII-À sa naissance, les pionniers de la nouvelle histoire ont voulu l'établir comme une véritable science sociale, par conséquent ouverte aux autres sciences humaines, en constant dialogue avec elles. Dans le champ de l'histoire sociale québécoise, ce genre d'échange, de réflexion conjointe au-delà des frontières disciplinaires, est très peu pratiqué. On pourrait dire que l'histoire sociale a maintenant assuré son territoire mais elle ne saurait vraiment le nommer.

Notre texte se veut un commentaire descriptif et une illustration de ces paradoxes, mais rien de plus. Les facteurs proprement explicatifs (par exemple, les conditions de production des connaissances historiques durant la période considérée, la dynamique spécifique de la science historique au Québec, les grandes idéologies qui ont prévalu, le silence - relatif - des intellectuels sur la place publique, etc.) n'y sont pas vraiment traités. Il y aurait là matière à un autre essai. Par ailleurs, étant donné l'espace qui nous était imparti, ce survol a dû nécessairement sacrifier bien des nuances et, en particulier, gommer d'importants éléments de diversité au sein de la science historique québécoise.

Toutefois, avant d'aborder directement la discussion des paradoxes, il est nécessaire de nous appuyer sur un aperçu diachronique de la science historique au Québec. Nous avons choisi de le construire en prenant à témoin les productions de la Revue d'histoire de l'Amérique française. Les données qui en ressortent serviront ensuite à nourrir notre analyse, en fournissant des fondements empiriques indispensables à un certain nombre d'énoncés (parties II et III). Notre démarche exige aussi de mettre en place une conception de l'histoire sociale, par rapport à laquelle nous tenterons de situer la pratique scientifique québécoise; cela nous amènera à rappeler les grandes orientations que poursuivait l'histoire sociale à l'origine (ce que nous avons appelé le «programme»). Enfin, il n'est nullement suggéré que les traits relevés en rapport avec la science historique québécoise lui soient exclusifs comme nous l'indiquerons, à l'occasion.

\section{I - APERÇU QUANTITATIF}

Afin de repérer certaines grandes tendances en cours au sein de la science historique québécoise, nous avons cru utile de prolonger la compilation effectuée il y a un quart de siècle par Fernand Harvey et 
Paul-André Linteau ${ }^{4}$. À cette fin, nous avons procédé 5 à un relevé des articles, notes de recherche et comptes rendus publiés dans la Revue d'histoire de l'Amérique française durant les 35 dernières années (1962-1996). Des compilations quinquennales ont été effectuées à l'aide d'une grille thématique comportant 34 entrées (Annexe A: Grille intégrale). Le caractère détaillé de la grille a été dicté par la grande variété des thèmes abordés dans la Revue et par la nécessité d'en fournir un aperçu qui soit le plus fidèle possible. Pour certaines analyses, il s'est toutefois révélé profitable d'en tirer une deuxième grille en procédant à des regroupements (Annexe B: Grille agrégée, 14 entrées). Les calculs ont porté à la fois sur le nombre de textes publiés et sur ce que nous avons appelé le nombre de mentions. Ce double compte s'est avéré nécessaire étant donné que certains textes pouvaient être associés à plus d'une rubrique dans la grille intégrale ${ }^{6}$. À moins d'avis contraire, nos commentaires porteront uniquement sur la statistique des mentions. Faute de place, les tableaux réalisés n'ont pu être reproduits ici ${ }^{7}$. Nous nous limiterons à un résumé des résultats les plus significatifs, utiles à notre propos. Au besoin, nous prolongerons nos commentaires en nous appuyant sur les compilations antérieures ${ }^{8}$.

Encore une remarque au sujet de nos relevés statistiques, pour signaler qu'ils ont donné lieu à deux séries de tableaux, l'une fondée sur les articles et notes de recherche (633 titres, 1030 mentions), l'autre sur les comptes rendus de volumes (1 838 titres, 2989 mentions). Les deux séries étant souvent discordantes, nous n'utilisons ici que la première. Ce choix a été dicté par le fait que, dans une proportion significative, les comptes rendus concernent des livres, des sujets

4. Fernand Harvey, Paul-André Linteau, «L'évolution de l'historiographie dans la "Revue d'histoire de l'Amérique française", 1947-1972», Revue d'histoire de l'Amérique française, 26,2 (septembre 1972): 163-183.

5. Avec l'assistance de Jeannette Larouche, que nous remercions vivement.

6. Par exemple, un ouvrage retraçant la vie d'un chef d'État relève de deux thèmes: Histoire politique et Biographie. De même, une étude sur la vie religieuse en milieu rural est associée à Histoire religieuse et à Société rurale, etc. Ce mode de compilation, qui n'entraîne aucun inconvénient sur le plan méthodologique, fournit un aperçu plus complet et plus précis de la pratique scientifique. Un procédé contraire amènerait en outre à faire des choix arbitraires et à biaiser le relevé. Classerait-on une étude sur un curé de campagne dans Histoire religieuse ou dans Société rurale? Dans un cas comme dans l'autre, on perd un important élément d'information et on ne sait justifier rigoureusement la décision qui est prise.

7. Ils ont été réunis dans Gérard Bouchard et Jeannette Larouche, Statistique des thèmes abordés dans la Revue d'Histoire de l'Amérique française (1962-1996). Document de l'IREP, II-C-253 (1997): 15.

8. Voir Fernand Harvey et Paul-André Linteau, loc. cit. Aussi: Jean-Paul Coupal, «Les dix dernières années de la Revue d'histoire de l'Amérique française, 1972-1981", Revue d'histoire de l'Amérique française, 36,4 (mars 1983): 553-567. 
et des auteurs non québécois9. Enfin, il aurait été utile de constituer une autre série statistique appuyée sur la production des mémoires et thèses; elle aurait peut-être donné des résultats un peu différents. Mais cette opération s'est heurtée à toutes sortes de difficultés et il a fallu $\mathrm{y}$ renoncer ${ }^{10}$. Nous nous en remettrons donc à un seul indicateur, celui des articles et notes. Dans le même ordre d'idées, il est certain que d'autres périodiques québécois auraient pu, avec profit, faire l'objet de compilations semblables. Nous nous en sommes cru dispensé, du fait que la $R H A F$ représente de loin la principale voie d'expression des historiens au Québec. Il est non moins vrai que ces historiens publient une partie de leurs articles à l'extérieur du Québec; mais cela ne nous paraît pas remettre en cause la représentativité de la $R H A F^{11}$.

Nous admettons d'emblée que ce genre de relevé thématique ne fournit qu'un aperçu partiel de la pratique scientifique, dont il ne reflète pas les problématiques, les démarches, les résultats. Il n'en fournit pas moins de précieuses indications sur la structure du champ (ex.: degré de fragmentation), sur les problèmes qui sont abordés et ceux qui sont délaissés (ou occultés?), sur le rapport à l'actuel, sur le degré d'ouverture aux réalités non québécoises (ex.: importance de l'histoire comparée), sur la place des autres sciences sociales, sur la conscience plus ou moins aiguë de son statut, de ses orientations, de ses méthodes, sur certaines spécificités par rapport à d'autres historiographies, etc. Ces résultats ne remplacent évidemment pas une véritable analyse de contenu; ils n'en éclairent pas moins des aspects importants de l'évolution et de la situation présente de l'histoire sociale.

Les données statistiques montrent d'abord que, durant la dernière période quinquennale (1992-1996), les quatre thèmes les plus fréquemment traités sont, dans l'ordre, la ville et l'industrie, la religion, la santé et les thèmes généraux d'histoire sociale ${ }^{12}$. Ils sont suivis, sur un pied d'égalité, de la famille, de la condition féminine et de l'histoire démographique. Cet ordre de fréquence est en lui-même révéla-

9. À remarquer qu'une certaine distorsion naît aussi de ce que certains articles ou notes sont écrits par des chercheurs non québécois. Mais ils sont peu fréquents et ils portent tous sur le Québec ou sur des populations francophones.

10. Répertoires inexistants, partiels ou dispersés; compilations par thèmes plutôt que par disciplines, etc. Pour un, le corpus réalisé par P. Aubin et L.-M. Côté (Bibliographie de l' histoire du Québec et du Canada) présentait deux inconvénients: il s'arrête en 1990 et les mémoires et thèses y sont regroupés avec les livres et articles (une seule année représente environ 5000 titres).

11. Cela dit, un biais assuré naît du fait que ce périodique reflète davantage la production francophone que la production anglophone, au sein de la science historique québécoise.

12. Sont regroupées dans ce sous-ensemble des études très diverses qui ont pour toile de fond des catégories, des classes ou des milieux sociaux (autres que les ouvriers ou les femmes, qui ont fait l'objet de rubriques séparées), des comportements collectifs, des rapports sociaux. 
teur, sans doute, mais il perd de sa signification lorsque l'on prend en compte l'allure très étalée de la distribution statistique. Ainsi, la rubrique la plus fréquente (ville et industrie) ne représente que $8,2 \%$ de l'ensemble des mentions et près de la moitié de celles-ci sont concentrées entre des fréquences de $2 \%-5 \%$. À l'autre extrémité, parmi les thèmes les plus délaissés, se trouvent la francophonie hors Québec, l'histoire générale, l'histoire militaire, la méthodologie, les Amérindiens. Nous y reviendrons.

Si l'on adopte maintenant une perspective diachronique, les phénomènes suivants ressortent:

- Deux thèmes ont connu une croissance forte; ce sont la ville et l'industrie (à partir de 1982-1986), la santé (surtout en 19921996). Ce résultat, tout comme celui qui suit, doit être interprété encore une fois à la lumière de l'importante dispersion des thèmes; ces hausses sont donc toutes relatives.

- Deux thèmes ont connu une croissance moyenne: la famille (à partir de 1977-1981), la condition féminine (à partir de 19871991), intégration sociale et marginalité (en 1992-1996).

- Thèmes en croissance faible ou très faible: histoire comparée (surtout en 1992-1996), histoire régionale (à partir de 1977-1981).

- Divers thèmes ont subi une décroissance plus ou moins forte; ce sont: l'histoire politique (à partir de 1972-1976), l'histoire militaire (à partir de 1967-1971), la biographie (à partir de 19721976), la société rurale et la colonisation (à partir de 1967-1971), la francophonie hors Québec (à partir de 1967-1971), l'éducation (à partir de 1976-1971).

- D'autres thèmes, à fréquence faible, n'ont pas bougé ou très peu; c'est le cas de l'ethnohistoire (autrefois: «folklore»), des arts et lettres, des Amérindiens, des mentalités, de la méthodologie, des sciences, des droits et libertés, des sports et loisirs, de l'histoire générale.

- Enfin, certains thèmes ont suivi des itinéraires plus compliqués, qui font alterner croissance et décroissance. Parmi ceux qui ont connu une hausse suivie d'un déclin, mentionnons l'histoire ouvrière (en baisse après 1977-1981), l'histoire économique (en baisse après 1972-1976), les thèmes généraux d'histoire sociale, l'histoire démographique, l'archivistique et l'histoire géographique/environnementale (en baisse après 1987-1991), l'historiographie et l'épistémologie (en baisse après 1972-1976). Soulignons en outre que quatre de ces thèmes (histoire ouvrière, archivistique, géographie/environnement, historiographie/épistémologie) avaient 
une fréquence très faible (1-2\%) en fin de période. Dans le sens contraire, deux thèmes se trouvaient en hausse durant la dernière période quinquennale, après avoir connu un déclin; ce sont la religion (forte baisse amorcée à partir de 1967-1971, croissance en 1992-1996) et l'histoire des idées (en baisse jusqu'en 1982-1986, en hausse par la suite). Enfin, la thématique nationale a connu l'itinéraire le plus brisé, soit: hausse jusqu'en 1967-1971, suivie d'un déclin, nouvelle hausse en 1977-1981, puis re-déclin important (1,5\% des mentions seulement en 1992-1996 $\left.{ }^{13}\right)$.

Nous avons calculé un dernier indice pour suivre à travers le temps l'évolution du caractère plus ou moins dispersé des thèmes, selon leur fréquence. Cet indice de fragmentation (If), calculé pour chaque période quinquennale, prend la forme d'un pourcentage; il a pour numérateur le nombre de mentions regroupées dans les dix thèmes les plus fréquents, et pour dénominateur le nombre total de mentions relevées dans l'ensemble des thèmes. Plus ses valeurs sont faibles, plus la fragmentation est donc accentuée. Le tableau 1 révèle qu'en 1962-1966, les 10 thèmes les plus fréquents réunissaient près de $80 \%$ des mentions; 25 ans plus tard, cette proportion tombait à $61,8 \%$. On reconnaît là l'effet de la spécialisation, de l'extension et de la diversification du territoire de l'historien. Ce dernier résultat reflète une perception qui était déjà répandue, mais il était utile de la valider empiriquement.

Sur certains autres points, ces statistiques n'offrent guère de surprises. L'essor général de l'histoire sociale était bien connu ${ }^{14}$, tout comme le déclin de la biographie ou de l'histoire militaire. Par contre, on se surprendra peut-être de l'émergence relativement tardive de la ville et de l'industrie, ou de la condition féminine. On constatera avec intérêt la croissance plutôt moyenne d'un thème comme la famille (compte tenu de la popularité de la microhistoire), la très faible fréquence du thème Amérindiens, le renouveau de l'histoire religieuse et de l'histoire des idées, la part relativement modeste des thèmes géné-

13. Il n'est pas toujours possible de comparer nos résultats avec ceux présentés par Fernand Harvey et Paul-André Linteau, loc. cit., et Jean-Paul Coupal, loc. cit. Ces auteurs ne semblent pas avoir tenu compte du problème des doubles mentions, ce qui entraîne des écarts dans les calculs. En outre, notre grille est plus détaillée et, même dans sa version agrégée, elle n'est pas totalement compatible avec celle utilisée par nos prédécesseurs. Des aires de comparaison subsistent néanmoins et permettent de constater un fort degré de concordance dans les tendances relevées. Dans les paragraphes qui précèdent, nous avons choisi de ne pas rapporter les résultats qui se trouvaient contredits par Harvey et Linteau ou par Coupal; ils sont, au demeurant, assez rares et concernent des aspects mineurs.

14. Cela, à l'échelle québécoise (Fernand Ouellet, loc. cit.) comme à l'échelle canadienne (David Gagan, H. E. Turner, "Social History in Canada: a Report on the "State of the Art"», Archivaria, 14 (été 1982): 27-52). 


\section{Tableau 1}

\section{Mesure de la fragmentation (lf) des thèmes dans la Revue d'histoire de l'Amérique française, 1962-1996 (proportion des mentions regroupées dans les 10 thèmes les plus fréquents)}

$\begin{array}{cccr}\text { Sous-périodes } & \begin{array}{c}\text { N. de mentions } \\ \text { regroupées dans les } \\ 10 \text { thèmes les plus } \\ \text { fréquents }\end{array} & \begin{array}{c}\text { N. total de mentions } \\ \text { dans les 33 thèmes } \\ \text { de la grille intégrale }\end{array} & \begin{array}{r}\text { Rapport de }(1) \\ (\%) \\ \text { (indice }\end{array} \\ 1962-1966 & 123 & 158 & \\ 1967-1971 & 134 & 164 & 78,3 \\ 1972-1976 & 86 & 137 & 61,7 \\ 1977-1981 & 94 & 158 & 69,8 \\ 1982-1986 & 103 & 153 & 67,3 \\ 1987-1991 & 85 & 129 & 65,9 \\ 1992-1996 & 81 & 131 & 61,8 \\ & & & 68,6\end{array}$

Note: Les calculs sont fondés sur les 1030 mentions dérivées des 633 articles et notes de recherche parus dans la revue entre 1962 et 1996.

raux d'histoire sociale. L'attention se porte aussi sur la désaffection de la thématique nationale et sur le déclin de l'histoire ouvrière, de l'histoire économique ${ }^{15}$, de l'historiographie/épistémologie et de la francophonie hors Québec - ce dernier point contrevenant à la vocation originelle de la revue. D'autres grands problèmes de l'heure, comme l'environnement, les droits et libertés ou l'économie sont très peu présents. Un commentaire plus détaillé (qui est impossible ici) ferait voir plusieurs autres phénomènes intéressants. Par exemple, l'essor de la thématique urbaine dissimule un important déséquilibre; la ville de Québec y est peu représentée, de même que les petites villes régiona-

15. Déjà signalée par quelques auteurs (Gilles Paquet, «Développement économique», dans Jacques Rouillard, dir., Guide d'histoire du Québec, du régime français à nos jours: bibliographie commentée (Montréal, Les Éditions du Méridien, nouvelle édition, revue et commentée, 1993), 147-148; J. E. Igartua, «L'histoire économique contemporaine: où sont passés les historiens?», dans Yves Roby et Nive Voisine, dir., Érudition, humanisme et savoir (Sainte-Foy, Les Presses de l'Université Laval, 1996), 153-168, Actes du colloque en l'honneur de Jean Hamelin). Paul-André Linteau offre un tableau moins sombre pour la période 1867-1929; voir «L'histoire économique du Québec de la période 1867-1929. Tendances récentes», dans Yves Roby et Nive Voisine, dir., Érudition, humanisme et savoir (Sainte-Foy, Les Presses de l'Université Laval, 1996), 131-152, Actes du colloque en l'honneur de Jean Hamelin. 
les (sauf Trois-Rivières), la plus grande partie des travaux portant sur Montréal ${ }^{16}$.

$\grave{A}$ une autre échelle, il importe de souligner les similitudes et les différences qui ressortent des trames québécoise et canadienne ${ }^{17}$. D'un côté comme de l'autre, on a assisté à un déclin de la thématique nationale et de l'histoire économique, parallèlement à une émergence de l'histoire des femmes. Mais l'essor de la société rurale, des études amérindiennes et des questions de droits et libertés dans l'historiographie canadienne n'a pas vraiment eu sa contrepartie à l'échelle québécoise. Ce trait mériterait d'être analysé de plus près, dans la mesure où ces thèmes (les deux derniers surtout) sont au cœur de l'actualité.

Tous ces résultats sont appuyés sur la statistique des articles et notes de recherche. Nous pensons que, dans les grandes lignes, ils n'auraient pas été bien différents si nous avions considéré les ouvrages publiés. Un examen rapide des principaux livres de la période (correspondant aux mêmes critères de sélection que les articles et notes de recherche) laisse en effet entrevoir les mêmes fluctuations, qu'il s'agisse, par exemple, de l'histoire ouvrière, de l'histoire des femmes ou de l'histoire urbaine.

\section{II - TRAJECTOIRES DE L'HISTOIRE SOCIALE QUÉBÉCOISE}

Pour mieux dégager la portée et l'intérêt des données qui précèdent, pour être en mesure aussi de mieux caractériser l'évolution et l'état de l'histoire sociale, il est nécessaire de rappeler d'abord ce qu'elle voulait être à l'origine. Dans une étude antérieure ${ }^{18}$, nous avons tenté de récapituler les idées fondatrices de l'histoire sociale, telles qu'elles ont été formulées entre 1940 et 1960 par des historiens français comme Marc Bloch, Lucien Febvre et Fernand Braudel, mais aussi Ernest Labrousse, Georges Lefebvre, Henri Pirenne (pour la Belgique), Jean Meuvret, Alphonse Dupront et quelques autres figures importantes que la mémoire officielle a un peu effacées. Nous avons retenu ces références francophones, de préférence à d'autres, d'abord à cause de l'influence qu'elles ont exercée un peu partout en Europe

16. En se reportant au survol détaillé publié par Fernand Ouellet en 1985, on perçoit bien l'importance des changements survenus depuis une douzaine d'années (et que rien ne laissait alors présager), tout particulièrement le déclin de l'histoire économique, de l'histoire ouvrière et de l'histoire-débat (critique, épistémologie, etc.).

17. Dans ce dernier cas, nous nous en remettons principalement à l'étude de Jean-Claude Robert, loc. cit.

18. Gérard Bouchard, «L'événement, l'individu, le récit: une nouvelle frontière pour l'histoire sociale?», dans Simon Langlois et Yves Martin, dir., L'horizon de la culture. Hommage à Fernand Dumont (Sainte-Foy, Les Presses de l'Université Laval/Institut québécois de recherche sur la culture, 1995), 299-319. 
et en Amérique (on y parle encore couramment de l'«École des Annales»), et parce que ce sont elles principalement qui ont présidé à l'introduction et à la diffusion de l'histoire sociale au Québec. Pensons à Fernand Ouellet, Jean Hamelin, Alfred Dubuc, Jean-Pierre Wallot, Louise Dechêne, Claude Galarneau, Pierre Savard et d'autres qui se sont faits les relais du nouveau paradigme. Pensons aussi à l'intensité des contacts et des échanges France/Québec par la voie des étudiants, des colloques, des professeurs invités, etc. ${ }^{19}$. On ne trouve rien de comparable entre le Québec et l'Angleterre, l'Allemagne ou les ÉtatsUnis. En conséquence, nous écartons de notre exposé ces références, qui sont également liées de près à la naissance de l'histoire sociale en Occident, mais qui ont exercé relativement peu d'influence au Québec, sauf peut-être auprès de collègues anglo-québécois ${ }^{20}$. Quoi qu'il en soit, on trouverait une très grande convergence si on confrontait systématiquement les orientations principales de ce que l'on a appelé l'histoire sociale en différents pays d'Europe et en Amérique. À cause de son influence prépondérante, il nous paraît donc logique de retenir la filière française, non pas comme modèle ou comme exemplaire, mais simplement comme terme de référence pour mieux situer ce que nous appelons l'histoire sociale au Québec. Notre choix apparaît d'autant plus justifié que les historiens québécois des dernières décennies n'ont pas essayé de récuser cette conception fondatrice pour en proposer une autre qui leur soit propre. Pour la plupart, ils s'y sont d'emblée référés, au moins implicitement.

En se référant aux principaux travaux des pionniers français (et tout particulièrement ceux de Bloch, Febvre et Braudel), on peut identifier une dizaine de propositions principales qui constituaient à l'origine ce que nous convenons d'appeler ici le programme de l'histoire sociale. Nous désignons par là les diverses orientations qui, d'un côté ou de l'autre, ont été mises de l'avant pour démarquer la nouvelle pratique scientifique de l'ancienne tradition de l'histoire dite positiviste, événementielle, narrative. Avec les années, ces propositions de réforme se sont diffusées au sein de la communauté historienne française, auprès de laquelle elles en sont venues à faire l'objet d'un large consensus, elles se sont peu à peu amalgamées dans ce qu'on a appelé l'histoire sociale, puis elles ont été diffusées au Québec. Rappelons brièvement ces propositions:

19. Pour les années 1960 et 1970 , ces échanges sont évoqués dans Alfred Dubuc, «L'influence de l'école des Annales au Québec», Revue d'histoire de l'Amérique française, 33,3 (décembre, 1979): 357-386.

20. Sur ce sujet, voir par exemple R. S. Neale, «Social History», dans Class in English History, 1680-1850 (Oxford, Basil Blackwell, 1981), Introduction, 1-17. 
1 -Étendre l'analyse à l'ensemble des acteurs, y compris les plus modestes, d'où une sensibilité particulière aux conditions de vie, aux comportements sociaux, aux classes.

2 -Derrière le fait ou l'événement, rechercher la structure, les articulations, les interactions, les fondements matériels, les rapports à l'espace. À partir de cet éclairage, recadrer les évolutions sociales, culturelles, politiques et autres.

3 -Dans un esprit de synthèse, porter attention à toutes les composantes du social pour les intégrer dans une dynamique d'ensemble (l'histoire, science de la synthèse, disait-on; ou encore: histoire sociale, histoire totale).

4 -Récuser les tentations déterministes et le mimétisme des sciences naturelles; sauvegarder la part d'imprévisible dans l'action collective (l'histoire, science des possibles).

5 -Établir l'histoire sociale comme étant «l'étude des hommes dans le temps», confrontée à un objet sans cesse changeant (l'histoire, science du changement).

6 -Chercher à capter la diversité des acteurs, des situations, des comportements, de façon à reproduire toute la complexité du social.

7 -Prendre conscience du caractère relatif de la connaissance historique, en tant qu'elle est non pas le reflet fidèle et absolu du réel mais seulement son approximation, au gré de constructions toujours à refaire, selon le principe de l'histoire-problème.

8 -Ouvrir la science historique aux autres sciences sociales, leur emprunter concepts, instruments et méthodes, entretenir avec elles un constant dialogue; conduire une réflexion constante sur sa démarche, ses procédés, ses interprétations.

9 -Réformer la tradition artisanale et individuelle de la recherche, au profit des collaborations interdisciplinaires et des travaux collectifs.

10 -Pratiquer sur une grande échelle l'enquête comparative afin d'acquérir une meilleure connaissance de l'objet social, sous toutes ses facettes et dans toute sa diversité.

11 -Étendre l'éventail des sources à exploiter, au-delà de l'archivistique traditionnelle, largement restreinte aux grands textes officiels ou institutionnels; moderniser les instruments et méthodes de collecte et de traitement des données.

On voit que l'histoire sociale voulait réformer la science historique à la fois sur le plan de la thématique (intérêt accru pour les rapports de classes, pour les inégalités, etc.) et sur le plan plus général 
de la démarche (l'attention portée au collectif et aux acteurs sociaux devait imprégner toutes les spécialités, tous les domaines du champ historique). Signalons par ailleurs que les onze propositions qui viennent d'être énoncées n'épuisent pas nécessairement l'ensemble des orientations suggérées. Elles pourraient aussi faire l'objet de formulations un peu différentes. Mais elles reproduisent certainement l'essentiel des conceptions de l'histoire sociale à sa naissance et elles suffisent à notre exposé ${ }^{21}$.

Comment se situe la pratique scientifique québécoise par rapport à chacune de ces propositions?

Dans l'appréciation des éléments de continuité et de différenciation, il importe d'abord de réaliser l'ampleur des perspectives qui étaient proposées et des ambitions affichées, ainsi que les énormes efforts exigés de la part de l'historien désireux de se conformer à cet idéal scientifique de l'histoire totale ou totalisante. Sans surprise, avec un recul d'un demi-siècle, on constate une distance importante entre ce programme et l'état présent de l'histoire sociale au Québec ${ }^{22}$. Les deux premiers objectifs ont été largement réalisés et la pratique de l'histoire - comme démarche scientifique - s'en est trouvée radicalement transformée. La sensibilité au social (et à l'espace) est un trait marquant des recherches historiques récentes ${ }^{23}$. À ce propos, il faut signaler l'essor spectaculaire de la microhistoire (l'ouvrage solide de Bettina Bradbury ${ }^{24}$, par exemple, en est une remarquable illustration), comme une sorte de corollaire d'une histoire non élitiste. L'attention portée aux acteurs anonymes, aux faits et gestes de leur vie quotidienne, invitait à un découpage très fin de l'objet social (l'univers domestique, la parenté, la culture matérielle, la sexualité, la vie conjugale, la fécondité, les âges de la vie, les métiers, les marginaux, etc.). De même (objectif 4), la science historique québécoise a récusé sans peine les tentations déterministes; en réalité, elle n'en a jamais guère éprouvé. Même, l'histoire quantitative proprement dite (au-delà des comptages sommaires) y a toujours occupé une place très marginale.

21. Hubert Watelet, de son côté, en a offert un compte rendu qui est très proche de nos énoncés; voir «Les rapports entre science et culture et les paradigmes du mouvement des Annales», dans Gérard Bouchard, dir., avec la collaboration de Serge Courville, La construction d'une culture. Le Québec et l'Amérique française (Sainte-Foy, Les Presses de l'Université Laval, 1993), 221-250.

22. Nous avons montré ailleurs (Gérard Bouchard, «L'événement, l'individu, le récit», loc. cit.) que l'héritage français lui-même a abandonné de larges segments du programme fondateur.

23. Pour ce qui est de l'histoire géographique proprement dite, elle est bien vivante comme on sait, grâce surtout aux chercheurs du Centre interuniversitaire d'études québécoises.

24. Bettina Bradbury, Familles ouvrières à Montréal: âge, genre et survie quotidienne pendant la phase d'industrialisation (Montréal, Boréal, 1995), 368. 
Il est bien connu que l'historien québécois (comme bien d'autres) n'est pas un adepte chevronné du chi carré et de la régression multiple. Pour certains, c'est le signe d'un heureux équilibre entre qualitatif et quantitatif. Pour d'autres, c'est une lacune scientifique importante qui voue parfois l'histoire sociale à l'approximation hasardeuse.

On observe également une grande continuité ou correspondance entre les objectifs $n^{\text {os }} 6,7,9,11$ et la pratique québécoise actuelle. La microhistoire est le laboratoire de la diversité et même de la singularité, les historiens ne cherchent plus à fixer le passé dans sa vérité intrinsèque et définitive, le modèle du chercheur solitaire et sédentaire est devenu minoritaire, l'enquête historique se nourrit désormais des témoignages les plus divers: des tessons de l'archéologue à la pellicule des premiers cinématographes.

Cela pour la continuité. En ce qui concerne les prises de distance, il faut d'abord souligner la place très restreinte dévolue à la synthèse. Quelques réalisations sont à signaler, certes, par exemple la série d'ouvrages sur l'histoire du catholicisme, publiés sous la direction de Nive Voisine. De très bons manuels ont aussi été produits (Linteau, Durocher et al., Young et Dickinson, et d'autres) qui récapitulent, principalement à partir des travaux publiés, les connaissances relatives à diverses périodes de l'histoire du Québec. À une autre échelle, les synthèses régionales produites par l'Institut québécois de recherche sur la culture appartiennent au même genre, avec un objectif plus poussé de vulgarisation et à une échelle spatiale plus restreinte. Mais ces manuels relèvent plus d'une histoire générale très sensible aux aspects sociaux que de l'histoire sociale totale (ou de la synthèse) telle que préconisée par les pionniers, c'est-à-dire un effort à nouveaux frais pour saisir en profondeur et dans toutes leurs interactions les composantes structurelles qui commandent le changement d'une société - ou d'un segment dans une société - durant une période donnée. L'histoire économique et sociale du Québec (Fernand Ouellet) et Habitants et marchands de Montréal (Louise Dechêne) sont deux exemples d'ouvrages (on en compte quelques autres, mais guère plus) qui, par leur démarche, ont le mieux concrétisé cet objectif global. Il appert que ce genre historique a été plus ou moins délaissé depuis une vingtaine d'années. N'est-il pas significatif que le dernier livre à avoir tenté ce type d'appréhension de la société québécoise sur une assez longue période soit venu d'un sociologue ${ }^{25}$ ? Enfin, à l'échelle régionale au sens large, on compte là encore quelques réali-

25. Fernand Dumont, Genèse de la société québécoise (Montréal, Boréal, 1993), 393. 
sations importantes, par exemple l'ouvrage de Paul-André Linteau ${ }^{26}$ sur la ville de Montréal ou celui de Louis Lavallée ${ }^{27}$ sur Laprairie; mais ce sont des exceptions. Il en est de même des grandes synthèses thématiques, où l'histoire des femmes fait presque cavalier seul ${ }^{28}$.

C'est évidemment la microhistoire qui a pris le relais, sa popularité croissante allant de pair avec la fragmentation des thèmes, déjà évoquée. Les objectifs de l'histoire sociale contenaient virtuellement ce principe d'émiettement de la recherche à micro-échelle. Saisir la diversité des situations et des acteurs, rendre compte de leurs comportements dans leur environnement proche, recréer les dynamiques fines qui commandent les grands rapports sociaux et le mouvement de la société, rechercher les structures complexes derrière les événements, reconstituer les principaux facteurs ou composantes en interaction: tout cela appelait une mobilisation considérable de données, de compétences et de moyens, d'où il a résulté, sans surprise, une sorte de division du travail. Mais il y a plus que cela; en principe, la microhistoire n'est pas incompatible avec l'histoire-synthèse (ou la macrohistoire) et rien n'empêchait d'instituer un aller et retour entre les deux.

Sur un autre plan, et comme l'a fait ressortir notre relevé thématique de la $R H A F$, des directions de recherche qui auraient dû être au cœur de la nouvelle histoire apparaissent plutôt négligées; c'est le cas aujourd'hui des rapports sociaux, de la structure de classes, des inégalités, de la mobilité sociale. L'histoire sociale se serait donc davantage imposée comme démarche générale que comme thématique particulière?

Il faut signaler aussi le peu de travaux réalisés sur le changement social comme tel. Sur ce plan, la réflexion théorique et méthodologique a peu avancé depuis vingt ans, soit depuis que le marxisme est tombé en défaveur. Rien, en effet, n'a vraiment pris le relais. En réalité, les historiens québécois, en majorité, semblent davantage préoccupés par le calendrier de l'évolution de leur société (rythmes et modalités de l'urbanisation, de l'industrialisation, de la laïcisation, de l'accès à la modernité, et le reste) et par le besoin de montrer que toutes ces transformations ont eu lieu en accord avec la conjoncture nord-américaine, sinon occidentale. En conséquence, on en sait beau-

26. Paul-André Linteau, Histoire de Montréal depuis la Confédération (Montréal, Boréal, 1992), 613 .

27. Louis Lavallée, La Prairie en Nouvelle-France, 1647-1760. Étude d'histoire sociale (Montréal, McGill-Queen's University Press, 1992), 301.

28. Par exemple: Collectif Clio, L'histoire des femmes au Québec depuis quatre siècles (Montréal, Quinze, 1982), 521. 
coup plus sur ces choses qui ont changé que sur la dynamique ellemême du changement, avec ses mécanismes déclencheurs, ses effets d'entraînement, ses blocages, ses calendriers sectoriels, ses interactions, ses complexités factorielles. Non sans relation avec ce dernier point peut-être, l'histoire proprement économique, comme avenue de spécialisation, a elle aussi déserté pratiquement l'atelier de l'historien.

Un autre objectif visé par l'histoire sociale concernait l'ouverture aux autres sciences sociales, le dialogue et le rapprochement interdisciplinaire. Évitons ici un malentendu. Les fréquentations entre spécialistes se sont beaucoup intensifiées depuis les années 1960 et les chercheurs sont mieux informés de ce qui se passe chez leurs voisins (grâce, notamment, aux colloques thématiques, aux équipes et aux centres de recherche, à la politique des bailleurs de fonds, à la réforme des programmes d'études avancées...). Mais à travers toute cette animation, les appartenances disciplinaires sont demeurées à peu près intactes et les champs traditionnels se sont perpétués. Par ailleurs, et surtout, la réflexion concertée et les débats sur les démarches, les méthodologies, les spécificités et les réorientations disciplinaires ont virtuellement disparu ${ }^{29}$.

Parmi les autres directions qui ont été peu explorées, il faut encore mentionner l'histoire comparative. On se rappellera que, pour Lucien Febvre et Marc Bloch notamment (comme pour d'autres historiens européens et américains), elle était appelée à s'établir comme un recours scientifique indispensable à l'historien, lui permettant de mieux relativiser et, en définitive, de mieux comprendre son objet en confrontant ses figures à toutes les autres possibles ${ }^{30}$. Ici, l'une des préoccupations principales était d'éviter le double piège des fausses singularités et des causalités artificielles. L'histoire sociale québécoise n'a pas suivi cette voie - sauf quelques exceptions encore une fois (dont fait partie le projet conjoint sur les sociétés rurales québécoise et française, en marche depuis vingt ans ${ }^{31}$ ). En fait, le principal souci comparatif qui s'est vraiment manifesté au cours des dernières décennies est né avec la conjoncture de la Révolution tranquille et l'essor du paradigme modernisateur (problématiques du retard, du rattrapage

29. Qu'on songe, par exemple, au débat que sociologues, anthropologues et historiens ont engagé dans les années 1960 autour du modèle de la «folk society» appliqué à la société québécoise (brièvement reconstitué dans Alfred Dubuc, loc. cit.).

30. Voir par exemple Marc Bloch, «Pour une histoire comparée des sociétés européennes", Revue de synthèse historique, 46: 15-50; La société féodale (Paris, A. Collin, 1939), 2 volumes.

31. Voir à ce propos le plaidoyer de Nadia Fahmy-Eid, «Histoire comparée, histoire plus vraie? Quelques balises et des promesses d'avenir», Revue de la Société historique du Canada, 7 (1997): 3-18. 
et de la normalité). Il a donné lieu à des comparaisons ad hoc (confrontation de statistiques sur un thème donné), mais non à des recherches systématiques qui cherchent à comprendre d'une manière concertée des dynamiques collectives parallèles. Par contre, on pourrait citer un certain nombre d'exemples de véritables recherches comparatives instaurées par des sociologues (notamment l'important projet dirigé par Simon Langlois à l'ex-Institut québécois de recherche sur la culture, ou encore les comparaisons Québec/Pologne auxquelles ont collaboré des sociologues de l'Université de Montréal - M. Fournier, G. Houle ${ }^{32}$...).

Enfin, il convient d'évoquer un dernier dossier qui, sans avoir figuré explicitement au programme fondateur, en faisait partie au moins à titre de corollaire: il s'agit de l'élaboration d'infrastructures de recherche pour la science historique. Certes, dans plusieurs domaines, celle-ci s'est munie de précieux instruments au Québec (inventaires, répertoires, dictionnaires, bibliographies, atlas, etc.). Mais elle a beaucoup moins investi dans les formes les plus modernes d'infrastructures, celles que les nouvelles technologies informatiques rendent possibles, notamment les bases de données (quel qu'en soit le format ou le modèle). De ce point de vue, et à l'image de plusieurs pays d'Occident, le Québec s'est montré bien timide, dans l'ensemble. Il n'est évidemment pas question ici de soutenir la comparaison avec les États-Unis, où ce genre d'entreprises a connu un grand essor ${ }^{33}$. Mais en se référant à des pays comme la Hollande, la Suède ou la Norvège, on voit bien que l'effort aurait pu être beaucoup plus considérable. Ainsi, si on passe en revue les trois principales banques de données informatisées présentement utilisées par les historiens au Québec, on constate que l'une a été mise sur pied par des démographes (Registre de la population du Québec ancien), une autre a été construite en dehors du circuit de la recherche institutionnelle (banque de données Parchemin, gérée par la Société Archiv-Histo), tandis que le fichier de population BALSAC a été financé dans sa plus grande partie par des bailleurs de fonds relevant de domaines autres que les sciences sociales et historiques. Ajoutons qu'on ne dispose toujours pas de grands recueils statistiques constitués à même les séries gouvernementales ou autres. De même, l'histoire sociale québécoise s'est très peu souciée de se munir d'assises méthodologiques aussi élémentaires que des

32. L'exemple des États-Unis et de l'Australie (pour s'en tenir à ces deux références) permet de réaliser à quel point l'historiographie québécoise a peu cultivé ce genre scientifique.

33. Pensons seulement à l'Inter-University Consortium for Political and Social Research, qui met présentement à la disposition des chercheurs quelques milliers de corpus de données informatisées. 
grilles de classement des données professionnelles ou des échelles de stratification sociale ${ }^{34}$.

Il est un peu surprenant qu'on n'ait pas davantage éprouvé le besoin de ce genre d'instrumentation qui fournit à la démarche scientifique des procédés d'objectivation et de systématisation, qui aménage aussi les conditions nécessaires à la critique et à la comparaison - d'autant plus étonnant en fait que la logique même de l'histoire sociale y poussait: d'un côté, l'étude de la masse des acteurs, du grand nombre, appelait une mobilisation importante de données (l'histoire dite sérielle n'en est qu'un exemple); de l'autre, des impératifs de rationalisation plaidaient en faveur d'une accumulation et d'une réutilisation (ou d'un recyclage) des données, à quoi se rattache l'idée d'infrastructure.

Ces remarques font voir la part de continuité et de discontinuité par rapport aux conceptions initiales de l'histoire sociale, telles qu'on peut les saisir à partir des sources françaises. Les éléments de discontinuité, tout particulièrement, montrent que l'histoire sociale a eu son histoire au Québec et qu'elle s'est donné des orientations, des figures, un style spécifiques. Mais cette histoire reste à écrire. De même, les spécificités de cette pratique scientifique ne sont pas bien connues parce que la plupart des auteurs ne sont pas enclins à commenter leur propre démarche (pour la justifier ou simplement pour la situer par rapport à d'autres) et qu'il n'existe guère de véritables discussions épistémologiques ou théoriques sur la discipline. Il s'ensuit une certaine confusion quant au statut et aux contenus de l'histoire sociale au Québec. Il se pourrait bien que le consensus qui semble régner présentement recèle en réalité une grande hétérogénéité qu'il serait utile de reconnaître plus à fond.

Encore une fois, le but du présent essai n'est pas de conduire cet examen détaillé des contenus; l'envergure de l'entreprise exigerait bien plus qu'un article. Nous visions simplement à mettre en forme quelques questions.

\section{III - DES PARADOXES}

À la lumière de tout ce qui précède, nous pouvons maintenant revenir sur nos sept paradoxes, pour essayer de faire le point. En ce qui concerne d'abord le programme des pionniers, il est clair que les

34. Pour une initiative dans cette direction, voir Gérard Bouchard, Tous les métiers du monde. Le traitement des données professionnelles en histoire sociale (Sainte-Foy, Les Presses de l'Université Laval, 1996), 323. Dans les années 1970, le Groupe de recherche sur la société montréalaise (animé par J.-P. Bernard, P.-A. Linteau et M. Grenon) avait lui aussi esquissé une démarche méthodologique de ce genre. 
idées fondatrices ont été très inégalement reproduites. Ce phénomène donne à penser que, dans le cours de son institutionnalisation, la notion d'histoire sociale a connu bien des glissements de contenu. Le paradoxe naît de ce que ces glissements ont sans doute résulté dans des conceptions scientifiques très variées, présentement dissimulées sous une fausse unanimité. On ressent ici le besoin d'une analyse critique qui ferait la part des choses.

Selon le deuxième paradoxe, l'histoire sociale québécoise s'est un peu détournée, depuis quelques années surtout, de certains problèmes qu'on s'attendrait à trouver au cœur de la réflexion et de la pratique scientifique. Nous pensons ici aux classes, aux rapports et mouvements sociaux, aux grands conflits collectifs. La statistique de la grille intégrale fait un peu illusion de ce point de vue; parce que les autres domaines de recherche y sont très émiettés, les thèmes généraux d'histoire sociale paraissent occuper une place plutôt confortable. Mais si on se reporte aux compilations fondées sur la grille agrégée, on observe que c'est en réalité l'histoire culturelle (histoire religieuse, histoire des idées, etc.), avec $22,6 \%$ des mentions, qui est la plus pratiquée, alors que les thèmes sociaux en général ne recueillent que $14,2 \%$ des mentions. À ce propos, il faut noter tout particulièrement la rareté des articles sur l'histoire ouvrière $(2,2 \%$ - grille intégrale) et sur la problématique des classes ou de la mobilité sociale ${ }^{35}$. Cet énoncé se vérifie encore davantage lorsqu'on se reporte aux ouvrages publiés sur le Québec depuis quinze ans: à part trois ou quatre livres (par ailleurs, importants, remarquables même ${ }^{36}$ ), ce champ est demeuré presque désert. Sous cet angle particulier, serions-nous en présence d'une histoire dite sociale qui, en réalité, le devient de moins en moins? On serait tenté de répondre oui si ce n'était des études sur la condition féminine. Celles-ci représentent en effet le principal élément de dynamisme et de renouvellement de la tradition la plus fondamentale de l'histoire sociale. Une réflexion sur les facteurs explicatifs devrait sans doute faire intervenir ici l'essor du néo-libéralisme; l'individualisme radical ne favorise-t-il pas une vision atomisée du social (ou du collectif) qui, à la limite, contient presque sa négation? De même, l'histoire de vie, indépendamment de sa très grande valeur intrinsèque sur le plan scientifique, doit une partie de sa popularité au nouvel essor de l'individualisme libéral.

35. Notons au passage que, même durant les années 1970, ce sont surtout les sociologues qui ont débattu ces questions au Québec, en s'appuyant principalement sur la théorie marxiste (Anne Légaré, «Heures et promesses d'un débat: les analyses des classes au Québec, 19601980», Les Cahiers du socialisme (1980): 60-85). À ce propos, voir aussi Jacques Rouillard, «Histoire sociale», dans Jacques Rouillard, dir., op. cit., 163-182.

36. Par exemple: José E. Igartua, Arvida au Saguenay. Naissance d' une ville industrielle (Montréal, McGill-Queen's University Press, 1996), 273. 
Le paradoxe élitiste est peut-être le plus insidieux: le praticien de l'histoire sociale ne sait pas bien se faire entendre de ceux-là même dont il se fait le porte-parole. En effet, en vertu de la réforme effectuée, l'histoire a cessé d'être un art et s'est constituée en science. Il est vrai que l'histoire sociale québécoise n'a jamais prétendu se faire scientifique à la manière des sciences naturelles ou comme l'économique prétend l'être, c'est-à-dire une science exacte qui décode la mécanique de son objet pour en énoncer les régularités, sinon les lois. Elle a seulement cessé d'être littéraire; elle ne fait plus guère de concessions à l'esthétique, se laissant tenter par l'énonciation froide qui est l'apanage des sciences dures. En s'institutionnalisant, l'histoire sociale s'est ainsi quelque peu marginalisée, le public s'alimentant de plus en plus auprès de médias autrement plus efficaces que sont la télévision, le cinéma, les musées, les reconstitutions de sites ou d'épisodes historiques, le roman. Il existe un écart important entre la production des connaissances en histoire sociale et leur diffusion dans le grand public. En conséquence, à cause de son langage et de ses procédés spécialisés, l'histoire sociale exerce relativement peu d'influence dans l'accréditation des représentations du passé au sein de la population; ses voisines (ou ses concurrentes?) y contribuent bien plus puissamment ${ }^{37}$.

Quant à l'histoire-synthèse, il est certain qu'elle doit faire appel à une mobilisation importante de connaissances monographiques. A cet égard, on pourrait trouver une sorte de logique dans le fait que des synthèses pionnières comme celles de Fernand Ouellet ${ }^{38}$ ou de Jean Hamelin et Yves Roby ${ }^{39}$ aient été suivies d'une myriade d'enquêtes relevant de la microhistoire: les ouvrages pionniers avaient en quelque sorte formulé les problèmes et fait voir les grandes avenues de recherche qu'il convenait désormais de parcourir patiemment, dans le détail. Mais il n'est pas sûr que les choses se soient passées ainsi. Car après s'être déployée dans la microhistoire, l'histoire sociale n'en est pas vraiment revenue. Une autre logique s'est peut-être combinée à la première: dans une certaine mesure, la microhistoire ne sert-elle pas aussi d'alibi à l'historien, qui a un peu tourné le dos aux grands dossiers de la société actuelle? Les praticiens de l'histoire sociale en savent de plus en plus sur des sujets de plus en plus pointus; un savoir

37. James Miller faisait le même constat pour le Canada anglophone, dans son adresse présidentielle à l'occasion du dernier congrès de la Société historique du Canada à St.John's (Terre-Neuve), en juin 1997.

38. Fernand Ouellet, Histoire économique et sociale du Québec, 1760-1850: structures et conjoncture (Montréal, Fides, 1966), 639.

39. Jean Hamelin et Yves Roby, Histoire économique du Québec, 1851-1896 (Montréal, Fides, 1971), 436. 
très riche se constitue grâce à des enquêtes patientes, ingénieuses et rigoureuses. Mais quelles sont les finalités de ces connaissances si elles ne sont pas conjuguées dans des représentations plus amples, au profit d'une véritable conscience historique, vivante et éclairée, en vue d'une meilleure compréhension de la société dans laquelle nous vivons? Si elle n'est pas ainsi raccordée à des problématiques macrosociales, la microhistoire ne risque-t-elle pas de reproduire à sa manière la richesse de diversité, l'attrait du singulier, le confort méthodologique ainsi que les limites épistémologiques de l'ancienne histoire événementielle et factuelle? La microdiversité laissée à ellemême ne devient-elle pas une sorte d'événementiel de l'histoire sociale?

Cette question est liée de près au paradoxe de l'actuel. Au Québec (mais ailleurs aussi ${ }^{40}$ ), la voix de l'historien (comme historien, comme scientifique) ne se fait plus guère entendre dans les débats sur les problèmes de l'heure. Assez curieusement, c'est depuis qu'il professe une articulation première à l'actuel qu'il s'est éloigné de l'actualité: l'historien part de l'actuel mais il n'y revient guère - l'histoire des femmes, encore une fois, faisant présentement figure d'exception. À titre d'exemple, on se reportera ici à l'époque des grandes controverses au sujet de la Cession et de ses conséquences sur l'évolution de la société francophone, ou sur le thème de la crise agricole dans le BasCanada au début du XIX ${ }^{\mathrm{e}}$ siècle (dossier plutôt technique en apparence, mais qui s'était chargé de divers enjeux culturels). Notre statistique des mentions selon la grille intégrale est éloquente à cet égard: la nation (au sens très large), l'ethnicité (notamment les rapports entre francophones et non-francophones), les Amérindiens (plus spécifiquement), l'environnement, de même que les droits et libertés comptent parmi les thèmes les moins fréquentés ${ }^{41}$. Or ce sont les plus chargés d'enjeux collectifs, et les plus controversés également. Visiblement, l'histoire sociale n'a pas toujours su résister à la tentation de découper son objet d'une manière qui la soustrait largement aux dissensions et à la contestation, au social en tant que contradictoire et conflictuel ${ }^{42}$.

40. Pour une, la situation française présente de nombreuses analogies avec le Québec; à ce sujet, voir par exemple Alain Caillé, La démission des clercs (Paris, Éditions La découverte, 1993), 297. Cet auteur propose une «repolitisation» des sciences sociales et historiques, accusées d'avoir divorcé d'avec le siècle, d'avoir renoncé à «penser leur temps».

41. On pourrait y ajouter les thèmes et questions reliés à la dépendance économique, à la vie démocratique et à la participation politique (à l'échelle locale comme à l'échelle nationale) ainsi qu'aux relations internationales.

42. Ce facteur est sans doute pour quelque chose dans la relative désaffection des historiens pour la période antérieure à 1867 (Allan Greer, «Canadian History: Ancient and Modern», Canadian Historical Review, 77,4 (décembre, 1996): 575-590) et ses dossiers chauds: le traitement réservé aux Amérindiens, la Cession, les Rébellions... 
Cependant, la recherche d'une fausse unanimité sous couvert de scientificité menace de conduire à long terme à une science historique très propre, assurément, mais inutile socialement - et peut-être stérile intellectuellement ${ }^{43}$.

Le débat sur Lionel Groulx peut servir ici d'exemple. Il a été lancé et alimenté principalement par des essayistes, des politologues et des littéraires, les historiens professionnels se tenant plutôt en touche (à de rares exceptions près $\left.{ }^{44}\right)$. Pourtant, ils auraient pu y apporter une autorité et une rigueur qui ont fait défaut au débat. Résultat: il est maintenant largement compromis, chacun ayant établi son siège sans qu'on n'y voie guère plus clair. Autre exemple: la Cession et ses répercussions. L'histoire sociale, qui a soigneusement évité ce dossier depuis 25 ans, va peut-être à nouveau se faire doubler. Car la préoccupation en rapport avec cet épisode historique n'a pas diminué. On le voit souvent présent dans les débats publics, soit en filigrane, soit d'une façon très explicite. Son histoire est refaite par des politiciens, des polémistes (et même des lieutenants-gouverneurs). Des politologues, des littéraires, des cinéastes le remettent à l'ordre du jour (Christian Dufour, Bernard Andrès, Jacques Godbout et quelques autres). Ne serait-il pas utile que les historiens refassent leurs comptes et communiquent ce qu'un quart de siècle d'histoire sociale a permis d'apprendre ou de mieux comprendre sur le sujet?

Les deux derniers paradoxes ont des résonances critiques et épistémologiques. Depuis Henri-Irénée Marrou, Raymond Aron et Adam Schaff ${ }^{45}$, les historiens savent que leur savoir n'est pas le miroir fidèle et immuable de la réalité; ce savoir est un construit qui diffère selon les points de vue et change avec le temps. De même, ils sont devenus conscients des limites de leurs procédés. Comment dès lors expliquer la rareté des écrits de nature épistémologique, théorique, historiographique, méthodologique ou même critique ${ }^{46}$ ? S'il est vrai que ce sont les apories, les contradictions, les divergences, la confrontation des thèses et les dialogues entre disciplines qui engendrent et reflètent

43. Jean-Marie Fecteau a formulé à ce sujet un constat qui s'apparente au nôtre. JeanMarie Fecteau, «La quête d'une histoire normale: réflexion sur les limites épistémologiques du "révisionnisme" au Québec», Bulletin d'histoire politique, 4,2 (hiver 1995): 31-38.

44. Par contre, les étudiants semblent s'y être montrés plus actifs.

45. Henri-Irénée Marrou, De la connaissance historique (Paris, Éditions du Seuil, 1954), 298; Raymond Aron, Introduction à la philosophie de l'histoire. Essai sur les limites de l'objectivité historique (Paris, Gallimard, 1948), 441; Adam Schaff, Histoire et vérité, essai sur l'objectivité de la connaissance historique (Paris, Anthropos, 1971), 352.

46. Il y aurait beaucoup à dire, notamment, sur l'insouciance méthodologique qui, le plus souvent, caractérise le discours critique sur la science historique québécoise. Soulignons seulement que, même si elle a énormément gagné sur le plan de la rigueur et de la diversité, elle s'est montrée peu novatrice sur le plan des méthodes. 
la vitalité et la richesse d'une science, alors il n'y a pas de doute, l'histoire sociale québécoise devrait susciter quelque inquiétude. On peut même craindre un peu qu'elle ne porte les vêtements d'un nouveau positivisme, beaucoup plus sophistiqué (et plus trompeur?) que l'ancien: des méthodologies qui procurent à leurs auteurs une grande sécurité, des reconstitutions empiriques très précises et détaillées, méritoires assurément, mais qui répondent à des questions très pointues, assez éloignées de la macrohistoire sociale et des urgences du présent. À divers égards, la très grande popularité qu'a connue l'étude des structures des ménages en Occident depuis 1970 est un exemple typique de ce style d'histoire.

\section{IV - DES DÉFIS, DES PRIORITÉS}

L'histoire sociale au Québec a donné lieu à de substantielles avancées scientifiques (se reporter notamment aux objectifs 1, 2, 6, 7, 9 et $11 \mathrm{du}$ «programme») et à quelques paradoxes. On pourrait à partir de là concevoir un autre programme pour les décennies à venir. Parmi les orientations et réorientations souhaitables, il y aurait de toute évidence un ordre de priorité à instaurer, à la lumière des quatre données suivantes: a) des éléments essentiels, substantiels, sont acquis; b) la situation québécoise présente d'importantes spécificités, ce qui appelle et justifie - une différenciation marquée par rapport aux modèles français et autres; c) parmi les propositions des fondateurs des années 1940-1950, certaines ont perdu de leur actualité, d'autres sont demeurées très pertinentes; d) il y a un déséquilibre à redresser qui appellerait une accentuation des contacts et des échanges entre historiens québécois (francophones principalement - on peut supposer que la remarque ne vaut pas pour nos collègues anglo-québécois) d'une part, et historiens anglophones (américains et canadiens, surtout), d'autre part.

Aussi, parmi les tâches pressantes, nous pensons qu'il faudrait consentir un effort accru pour mettre en place des infrastructures de recherche (méthodologies, banques de données et autres) et des dispositifs pour en assurer efficacement la gestion. Il faut le répéter: l'histoire sociale n'a toujours pas les moyens de ses ambitions (et elle n'a pas toujours non plus la patience de ses moyens). Elle doit en outre se constituer davantage en science de la synthèse, ce qui invite nullement à délaisser la microhistoire, mais à mieux la raccorder à la macrohistoire, en réintégrant pleinement dans ses analyses l'État, la nation, les classes sociales, le capitalisme, les rapports de domination 
et d'exploitation, etc. ${ }^{47}$. Sur ce chemin, la science historique retrouvera toute la problématique des rapports, des conflits et des mouvements sociaux, celle des inégalités aussi, et de la mobilité sociale. Le problème du changement s'y greffe de près, non pas seulement comme exercice de périodisation, mais comme analyse de la structure sociale en tant que produit précaire et instable de tensions et contradictions, arbitrées par des facteurs internes et externes. À ces conditions seulement, l'histoire sociale se posera comme véritable science de l'actuel, capable de projeter sur les grands problèmes de l'heure des aperçus éclairants - et sans se dénaturer comme discipline scientifique. Il paraît hautement souhaitable aussi que la science historique québécoise accède à une conscience critique plus vive à l'égard de ses pratiques, de son propre passé et de son objet, ce qui devrait du même coup la rendre plus curieuse des autres sciences sociales. Le discours historiographique n'étant pas innocent, il devrait sans cesse instruire lui-même son procès. Enfin, il faut de toute évidence plaider vigoureusement pour les études comparatives, dans la mesure où la comparaison est un puissant procédé de dépaysement, de confrontation, de validation et d'objectivation.

On nous permettra de signaler (trop) rapidement trois autres directions qui, selon nous, devraient davantage solliciter l'attention des spécialistes de l'histoire sociale (et de la science historique en général). C'est d'abord la nécessité d'accroître sa participation dans l'entreprise générale de construction mémorielle; n'est-il pas utile que l'imaginaire collectif soit le plus possible imprégné des contenus les plus rigoureux? Cela exige que soient en partie repensés les procédés habituels de diffusion des connaissances savantes ${ }^{48}$. En deuxième lieu, autant, d'un côté, l'histoire sociale doit se donner des infrastructures et des méthodologies sophistiquées, autant, d'un autre côté, elle doit se soucier d'intégrer d'une manière ou d'une autre dans ses analyses l'individu, l'événement, le récit: si, comme la plupart des historiens l'affirment implicitement, le social n'est pas un système fermé, autodéterminé, il se déploie dans la durée et offre donc prise à une forme de narration. Cet énoncé marque, notamment, la place légitime mais laissée un peu vacante depuis quelques décennies - de la biographie dans l'histoire sociale. Enfin, armée de son savoir spécifique, celle-ci doit renouer pleinement avec une grande tradition critique de l'historiographie occidentale: critique des pouvoirs et de leurs straté-

47. À ce propos, voir l'excellent texte de Tamara K. Hareven, «What Difference Does It Make?», Social Science History, 20,3 (automne 1996): 317-344.

48. Le monde muséal, qui a fait depuis 25 ans de gros efforts pour renouveler ses procédés, pourrait ici servir d'exemple. 
gies; critique des idéologies, des représentations collectives; critique des machinations, des faux-semblants, des stéréotypes, des tabous de la pensée et de l'action. En ce sens, ne conviendrait-il pas que l'histoire soit aussi la science par où, de temps à autre, le scandale arrive ${ }^{49}$ ?

Cela étant dit, des événements récents dans le champ de l'histoire québécoise annoncent des changements dans le sens suggéré ici. Le nouveau Bulletin d'histoire politique représente une histoire plus combative, plus près des débats actuels (thèmes de numéros récents: l'enseignement de l'histoire nationale, les Anglo-Québécois, une nouvelle histoire du Québec, etc.). Les critiques adressées par Ronald Rudin à ce qu'il a appelé l'histoire révisionniste ont lancé un vrai débat historiographique. La Revue d'histoire de l'Amérique française a ellemême ouvert ses pages à quelques échanges critiques et vient d'inscrire une rubrique «Débat» à son format; en outre, l'IHAF a choisi la pratique de l'histoire comme thème de son congrès à l'automne de $1997^{50}$. Les Cahiers d'histoire du Québec ont aussi introduit un élément de diversification dans le paysage scientifique. La discussion en cours sur l'enseignement de l'histoire nationale rejoint un important sujet d'actualité. Enfin, le genre biographique vient d'être remis à l'honneur, notamment avec l'ouvrage d'Yvan Lamonde ${ }^{51}$, et l'histoire comparative fait depuis quelques années l'objet d'une programmation à long terme à l'IREP (Institut interuniversitaire de recherches sur les populations), autour de la problématique des collectivités neuves et cultures fondatrices.

Il reste beaucoup à faire, certes. Mais ce cinquantième anniversaire de la Revue d'histoire de l'Amérique française pourrait être l'occasion d'une prise de conscience et l'amorce d'une relance de l'histoire sociale au Québec.

49. Certains signes donnent à croire que, sous ce rapport, elle a pleinement joué son rôle au Canada anglais... Voir l'attaque dirigée par Michael Bliss, «Privatizing the Mind: the Sundering of Canadian History, the Sundering of Canada», Revue d'études canadiennes/Journal of Canadian Studies, 26,4 (1991): 5-17, contre l'histoire sociale, accusée d'avoir été un agent corrosif de l'unité nationale et de l'identité canadienne. thème?

50. L'institut ne pourrait-il pas, lors de ses congrès, réserver quelques sessions à ce

51. Yvan Lamonde, Louis-Antoine Dessaulles, 1818-1895: un seigneur libéral et anticlérical (Montréal, Fides, 1994), 369. 


\section{Annexe A}

\section{Thèmes de la grille intégrale}

1 - Thèmes généraux d'histoire sociale

2 - Intégration, marginalité, "pathologie» sociale

3 - Histoire ouvrière

4 - Ville, industrie

5 - Agriculture, société rurale

6 - Famille

7 - Condition féminine

8 - État, politique

9 - Histoire militaire

10 - Géographie/environnement

11 - Histoire démographique

12 - Éducation, alphabétisation

13 - Religion

14 - Santé, soins, maladies

15 - Ethnohistoire

16 - Science et technologie

17 - Biographie

18 - Histoire comparée

19 - Histoire économique

20 - Archivistique, sources, répertoires

21 - Histoire des idées

22 - Nation, ethnicité, langue

23 - Peuplement, colonisation

24 - Amérindiens

25 - Droits et libertés

26 - Arts et lettres

27 - Mentalités

28 - Francophonie hors Québec

29 - Sports et loisirs

30 - Régions

31 - Histoire générale

32 - Historiographie, épistémologie

33 - Méthodologie

34 - Autres, divers 


\section{Annexe B \\ Thèmes de la liste agrégée}

I - Thèmes généraux d'histoire sociale ${ }^{\mathrm{a}}$

II - Organisation, intégration, «pathologie sociale»

III - Géographie/environnement

IV - Démographie, santé

$\mathrm{V}$ - Monde rural

VI - Économie urbaine et industrielle

VII - État, politique, armée, justice

VIII - $\quad$ Nation, ethnicité, langue, Amérindiens

IX - Science et technologie

$\mathrm{X} \quad-\quad$ Biographie

XI - Histoire culturelle (éducation, religion, idées, etc.)

XII - Histoire comparée

XIII - Épistémologie, historiographie, méthodologie, archivistique...

XIV - Autres, divers ${ }^{\mathrm{b}}$

a Intègre Condition féminine et Histoire ouvrière.

b Intègre aussi quelques rubriques (à faibles fréquences) de la grille intégrale (Histoire générale, Sports et loisirs...). 
